\title{
IS-2: Network Structure and Dynamics of Biological Systems
}

\author{
Deena Schmidt \\ Department of Mathematics and Statistics, University of Nevada Reno, Reno, NV 89557 \\ drschmidt@unr.edu
}

Many biological systems in nature can be represented as a dynamic model on a network. Examples include gene regulatory systems, neuronal networks, food webs, epidemics spreading within populations, social networks, and many others. A fundamental question when studying biological processes represented as dynamic models on networks is to what extent the network structure is contributing to the observed dynamics. In other words, how does network connectivity affect a dynamic model on a network? I will introduce a variety of network topologies and discuss biologically-inspired dynamic models that evolve on such networks. I will also present several open problems in this area geared for undergraduate research. 